\title{
Superfluid density versus transition temperature in a layered organic superconductor $\kappa-(\mathrm{BEDT}-\mathrm{TTF})_{2} \mathrm{Cu}\left[\mathrm{N}(\mathrm{CN})_{2}\right] \mathrm{Br}$ under pressure
}

\author{
Kodai Wakamatsu $\odot,{ }^{*}$ Kazuya Miyagawa $\odot$, and Kazushi Kanoda $\odot^{\dagger}$ \\ Department of Applied Physics, University of Tokyo, Bunkyo-ku, Tokyo 113-8656, Japan
}

(Received 31 July 2020; accepted 15 September 2020; published 1 October 2020)

\begin{abstract}
A deficiency in the superfluid density in overdoping cuprates is an unconventional feature of keen interest in superconductivity with an electron correlation and, likewise, it was previously suggested that pressure, which weakens the electron correlation, induces an increased deficiency in the superfluid density in an organic superconductor with a fixed band filling. Here, we report our magnetic penetration depth measurements of a layered organic superconductor situated near a Mott transition under various pressures to revisit the issue of superfluid density. The analyses of the results find no meaningful variation in superfluid density with increasing pressure, which greatly suppresses the superconducting transition temperature.
\end{abstract}

DOI: 10.1103/PhysRevResearch.2.043008

\section{INTRODUCTION}

Superconductivity emerging in correlated electron systems exhibited various unconventional behaviors out of the standard BCS framework [1]. The cuprates harbor a range of phenomena of keen interest particularly in the underdoped region where electron correlation is enormously enhanced [2]. Although superconductivity in overdoped cuprates appears more conventional, several issues are yet to be solved, among which is the puzzle of missing superfluid density [3-11]; doping which generates mobile carriers contrarily leads to a reduction in the superfluid density $n_{\mathrm{s}}$ or in that divided by the effective mass $n_{\mathrm{s}} / m^{*}$ along with the transition temperature $T_{\mathrm{c}}$, in stark contrast to the underdoped region where $n_{\mathrm{s}} / \mathrm{m}^{*}$ and $T_{\mathrm{c}}$ increase in parallel with carrier doping [3]. The puzzle of the missing superfluid density or spectral weight arguably invokes phase separation [4], a disorder effect or pair breaking [5,7], and quantum critical fluctuations $[9,11]$.

Alternatively, the electron correlation can be varied by pressure that widens the electronic band while its filling is fixed. This method is particularly effective for organic crystals hosting highly compressible lattices [12]. The bandwidth in organic conductors is roughly an order of magnitude narrower than in inorganic materials such as highly correlated transition-metal oxides, making electrons more highly correlated in the former. The representative is a family of layered compounds, $\kappa$-(BEDT-TTF $)_{2} X$, in which BEDT-TTF molecules constitute two-dimensional conducting layers, as shown in Fig. 1(a), where BEDT-TTF denotes

\footnotetext{
*Corresponding author: wakamatsu@mdf2.t.u-tokyo.ac.jp

${ }^{\dagger}$ Corresponding author: kanoda@ap.t.u-tokyo.ac.jp
}

Published by the American Physical Society under the terms of the Creative Commons Attribution 4.0 International license. Further distribution of this work must maintain attribution to the author(s) and the published article's title, journal citation, and DOI. bis(ethylenedithio)tetrathiafulvalene and $X$ stands for an anion species [13,14]. Indeed, they include Mott insulators and remarkably, in the vicinity, superconductivity appears, which is suggested to be of $d$-wave nature by many experimental and theoretical studies [15-17]. The cuprates and organics share the common feature that superconductivity emerges from single-band Mott insulators by doping or pressure [18-20], and thus it is interesting to see comparatively the $n_{\mathrm{s}}$ behaviors of the doping-controlled cuprates and bandwidth-controlled organics.

Notably, $n_{\mathrm{s}}$ in $\kappa$-(BEDT-TTF) ${ }_{2} \mathrm{Cu}(\mathrm{NCS})_{2}$ (abbreviated as $\kappa$-NCS hereafter), which is renowned as the first over-10-K organic superconductor, was reported to decrease along with $T_{\mathrm{c}}$ by pressure in a study of the magnetic penetration depth $\lambda$, which gives the $n_{\mathrm{s}}$ through $1 / \lambda^{2} \propto n_{\mathrm{s}} / m^{*}$ in the London model [21]. This possibly suggests that the overdoped cuprates and pressurized organics have commonalities regarding the puzzling behavior of the superfluid density. In the present paper, to examine whether the pressure dependence of $n_{\mathrm{s}}$ found in $\kappa$-NCS holds in a different system, we have studied the pressure dependence of the superfluid density in an analogous material, $\kappa$-(BEDT-TTF $)_{2} \mathrm{Cu}\left[\mathrm{N}(\mathrm{CN})_{2}\right] \mathrm{Br}(\mathrm{ab}-$ breviated as $\kappa$-Br hereafter), which also resides near the Mott critical pressure and $T_{\mathrm{c}}$ is about $11.6 \mathrm{~K}$. We have found no meaningful suppression in $n_{\mathrm{s}}$ while $T_{\mathrm{c}}$ is reduced by $60 \%$ from the ambient-pressure value.

\section{EXPERIMENT}

Single crystals of $\kappa-\mathrm{Br}$ were prepared by the conventional electrochemical method [22]. The magnetic penetration depth $\lambda$ was determined by the so-called reversible magnetization method, in which the superconducting diamagnetism in the mixed state is related to $\lambda$, which characterizes the fielddecay length of penetrating vortices in the mixed state, and therefore the strength of intervortex repulsion. In the magnetization experiments, we stacked two blocklike crystals of $0.9 \times 1.2 \times 0.7$ and $0.9 \times 0.9 \times 0.7 \mathrm{~mm}^{3}$ in dimensions and 

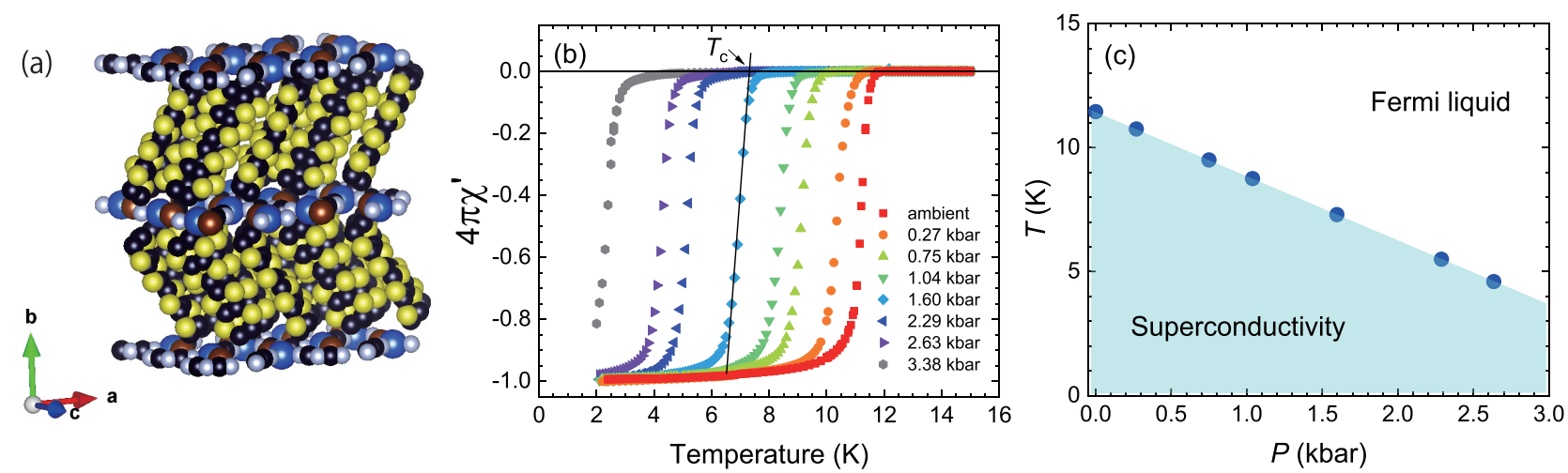

FIG. 1. (a) Crystal structure of $\kappa$-Br. A quasi-two-dimensional structure is composed of conducting BEDT-TTF layers and insulating anion layers. (b) Temperature dependence of ac susceptibility of $\kappa$-Br at ambient and applied pressures. An ac field of 1.0 Oe in amplitude and $10 \mathrm{~Hz}$ in frequency, which is low enough to avoid the shielding effect of the pressure cell, was applied perpendicular to the conducting layers. The saturation of $4 \pi \chi^{\prime}$ at low temperatures is regarded to give perfect diamagnetism and its value is taken as -1 . The superconducting transition temperature $T_{\mathrm{c}}$ was determined by the linear extrapolation of the steeply varying part of the ac susceptibility below $T_{\mathrm{c}}$, as shown in (b). (c) Temperature-pressure phase diagram of $\kappa-\mathrm{Br}$. Transition temperature $T_{\mathrm{c}}$ decreases as the pressure increases. The pressure values were estimated from the reported pressure derivative of $T_{\mathrm{c}},-2.6 \mathrm{~K} / \mathrm{kbar}$.

of $2.16 \mathrm{mg}$ in total weight to gain the magnetization signals. The magnetization was measured with a Quantum Design superconducting quantum interference device (SQUID) magnetometer (MPMS-XL) under upward and downward field sweeps up to $5 \mathrm{~T}$ at each measured temperature and pressure. For pressure application, the crystals were mounted in a clamp-type pressure cell made from $\mathrm{BeCu}$ with two quartz rods used as spacers [23]. We used Daphne oil 7373 as a pressure-transmitting media. The crystals in the pressure cell were aligned such that the direction of the applied field is normal to the conducting plane. We confirmed that the $\mathrm{BeCu}$ pressure cell used does not contain impurity magnetism nor superconductivity influential to the magnetization measurements. The internal pressure in the cell was estimated with reference to the reported pressure dependence of $T_{\mathrm{c}}$ of $\kappa-\mathrm{Br}$, which ranges from -2.4 to $-2.8 \mathrm{~K} / \mathrm{kbar}$ [24-26]; here, we adopted the median value, $-2.6 \mathrm{~K} / \mathrm{kbar}$. It is known that the cooling speed of $\kappa$-Br affects properties such as $T_{\mathrm{c}}$ and the electron mean free path most likely due to a cooling rate-dependent disorder of the terminal ethylene groups in BEDT-TTF [27-30]. To minimize this effect, we cooled the sample at rates of less than $0.5 \mathrm{~K} / \mathrm{min}$ from ambient temperature down to $10 \mathrm{~K}$. The superconducting transition under pressure was examined by ac susceptibility measurements.

\section{RESULTS AND DISCUSSION}

\section{A. Magnetization measurements}

Figure 1(b) shows the temperature dependence of ac susceptibility for an ac field of 1.0 Oe in amplitude and $10 \mathrm{~Hz}$ in frequency at the pressures investigated. The transition to perfect diamagnetism remains sharp even under pressure, ensuring the homogeneity of superconductivity over the investigated pressures. The onset of diamagnetism, $T_{\mathrm{c}}$, of $11.5 \mathrm{~K}$ at ambient pressure reproduces the reported value and monotonously decreases with increasing pressure as shown in Fig. $1(\mathrm{c}) ; T_{\mathrm{c}}$ is reduced to $4.6 \mathrm{~K}$ ( $40 \%$ of the pristine value) at 2.6 kbar.
The field dependence of magnetization $M$ for strong type-II superconductors $(\kappa=\lambda / \xi \gg 1$ with $\xi$ the in-plane superconducting coherence length) is given by

$$
-4 \pi M=\frac{\alpha \phi_{0}}{8 \pi \lambda^{2}} \ln \left(\frac{\beta H_{\mathrm{c} 2}}{H}\right)
$$

for a field range of $H_{\mathrm{c} 1} \ll H_{\text {irr }}<H \ll H_{\mathrm{c} 2}\left(H_{\text {irr }}\right.$ : irreversible magnetic field above which the magnetization is reversible; $H_{\mathrm{c} 2}$ : upper critical field; $\phi_{0}$ : flux quantum; $\alpha$ : core contribution factor; $\beta$ : a constant of order of unity) [31]. In this field range, vortices are free from collective pinning and magnetization is reversible against field sweeps. Equation (1) suggests that $d M / d(\ln H)$ gives an estimate of $\lambda$. As the correction factor $\alpha$ from the London model, which does not consider the vortex core contribution, we adopted the value $\alpha=0.77$, calculated in Ref. [32].

The magnetization in the present experimental setup includes the contributions of the sample, pressure cell, and their surroundings such as oil, and is spatially inhomogeneous along the scanning direction in the measurement; so, the SQUID signal form was not the ideal one expected for a pointlike magnetization and the conventional background subtraction procedure is not effective, in which the magnetization of the pressure-cell system without the sample is separately measured. To overcome this difficulty, we recorded the entire SQUID signals (raw wave-form data) acquired during the scanning for all of the runs and performed the subtraction between them at the same temperature and magnetic field. Another problem was the reproducibility of the background; after the sample was removed from the pressure cell for the background measurement, it was difficult to reassemble the pressure cell system so as to reproduce the background magnetization before reassembling with a better precision than the small superconducting diamagnetic signals in the high-field region. To avoid this complication, we used the wave-form data at a high pressure, $3.4 \mathrm{kbar}$, where superconductivity in $\kappa-\mathrm{Br}$ is suppressed and the magnetization of the normal state of $\kappa-\mathrm{Br}$ (at most $-10^{-6}$ emu at $1 \mathrm{~T}$ ) is small enough, as the background signal that is acquired without removing the 

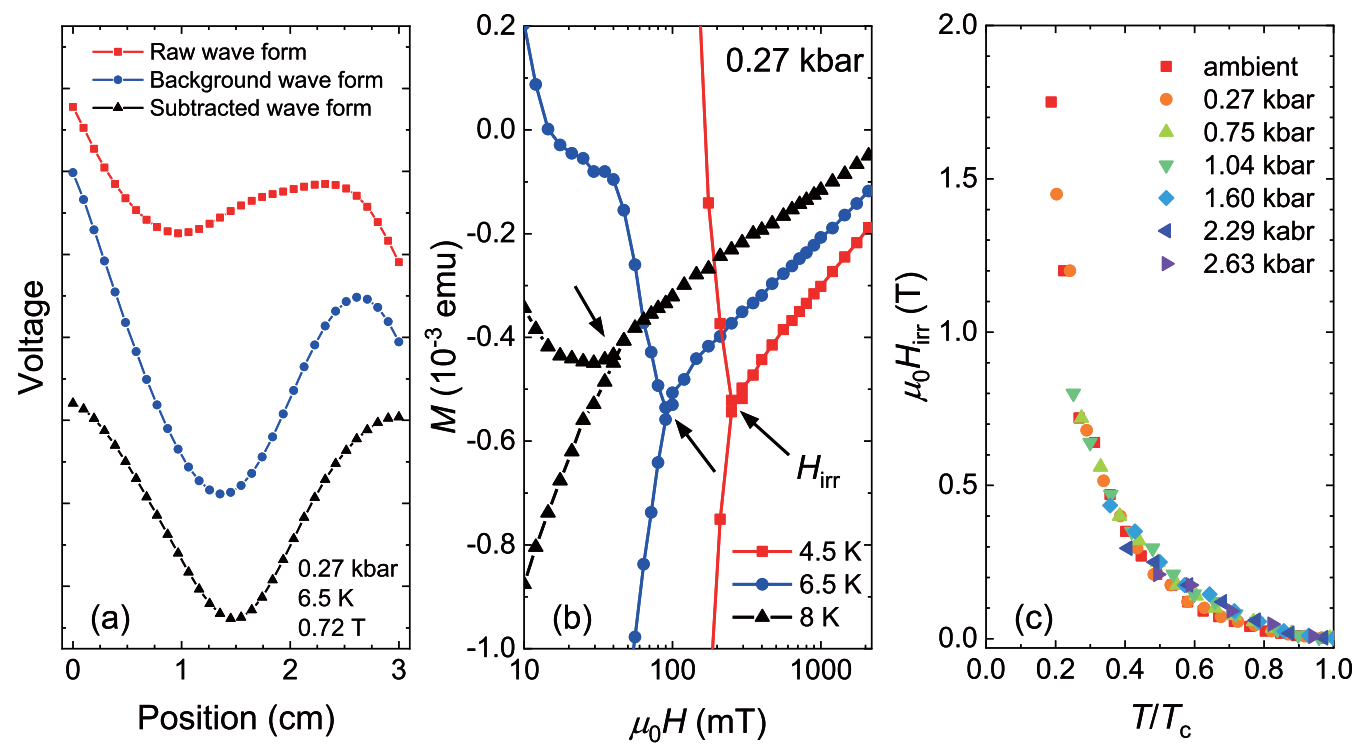

FIG. 2. (a) SQUID scanning wave forms in magnetization measurements of $\kappa$-Br. The horizontal axis shows the sample position inside the SQUID pickup coil during the scans. The red squares, blue diamonds, and black triangles indicate a wave form with the contribution of superconducting diamagnetism, a high-pressure wave form without it, and a subtracted wave form giving the sample magnetization, respectively (the former two wave forms are inverted vertically). (b) Magnetization vs applied magnetic field at 0.27 kbar. Each data point in the magnetization curves was obtained from the background-subtracted wave form exemplified in (a). The arrows point to the $H_{\text {irr }}$, above which the magnetization is free from hysteresis. (c) Irreversible magnetic field vs reduced temperature $T / T_{\mathrm{c}}$.

sample from the cell. Besides, we note that the sample volume contracts under pressure, albeit only slightly, e.g., by $2 \%$ at $2.6 \mathrm{kbar}$ [33], so, we corrected this effect in the following results, although practically not influentially.

Figure 2(a) shows three typical SQUID response signals, which include a wave form with the contribution of superconducting diamagnetism, a high-pressure wave form without it, and a subtracted wave form, which is found to successfully take an expected shape and thus is converted to the sample magnetization by fits using the formula given in Ref. [34]. We performed this background correction procedure for all data points presented here. Figure 2(b) thus shows the deduced magnetizations, which clearly identify the irreversibility field $H_{\text {irr }}$, above which the magnetization is free from hysteresis. The $H_{\text {irr }}$ rapidly rises with decreasing temperatures at each pressure and appears to fall into a common curve when the temperature is normalized to $T_{\mathrm{c}}$, as seen in Fig. 2(c). The magnetizations in the reversible regions are plotted against $\ln H$ in Fig. 3; the magnetization data for all pressures studied are shown in Fig. 3. They are well approximated by linear relations for every temperature and pressure investigated, ensuring the prerequisite for deducing $\lambda$ from magnetization through Eq. (1).

\section{B. Magnetic penetration depth}

The $1 / \lambda^{2}$ values determined by the slopes of the observed linearities through Eq. (1) are plotted with respect to temperature at each pressure studied in Fig. 4. The $1 / \lambda^{2}$ increases on cooling and keeps increasing quasilinearly even at the lowesttemperature regions, reminiscent of nodal superconductivity, however, the closer examination of which requires $1 / \lambda^{2}$ data at lower temperatures. The present work does not discuss the symmetry of the Cooper pair but instead sees the pressure dependence of the low-temperature limit, $1 / \lambda(0)^{2}$, giving the superfluid density. We note that the temperature profile of $1 / \lambda^{2}$ is pressure independent, as shown in Fig. 4, which ensures the reliability of the present analysis. To evaluate the $1 / \lambda(0)^{2}$ values at each pressure, we fitted the $1 / \lambda(T)^{2}$ data by the formula $1 / \lambda(T)^{2}=1 / \lambda(0)^{2} \times\left[1-\left(T / T_{\mathrm{c}}\right)^{p}\right]$ with the exponent $p$ fixed at $2,1.75$, and $4 / 3$. It is known that $p=2$ and $p=4 / 3$ give good approximations for the $s$-wave and $d$-wave cases, respectively, [35] and $p=1.75$ was the average of the $p$ values fitting the $1 / \lambda(T)^{2}$ data at every pressure. As seen below, the choice of the $p$ value makes no appreciable difference to the $1 / \lambda(0)^{2}$ values and its pressure dependence (as seen later in Fig. 5). The value of $\lambda(0)$ at ambient pressure is $490-520 \mathrm{~nm}$, which is in a range of the previously reported values, $570 \mathrm{~nm}$ [29], $464 \mathrm{~nm}$ [30], and $650 \mathrm{~nm}$ [36].

With a finite electron mean free path $l$, the observed penetration depth $\lambda$ is related to the London penetration depth $\lambda_{\mathrm{L}}\left[=\left(\mu_{0} n_{\mathrm{s}} e^{2} / m^{*}\right)^{-1 / 2}\right]$ by $\lambda^{2}=\lambda_{\mathrm{L}}^{2}(1+\xi / l)$ for a situation of $\lambda>l>\xi$ [37]. In $\kappa-\mathrm{Br}$, the experimental values of $l$ and $\xi_{\text {expt }}$ are 26-38 nm [28,38,39] and 2.4-3.7 nm [40,41] at ambient pressure, respectively. Employing the median values of $l=32 \mathrm{~nm}$ and $\xi_{\text {expt }}=3.1 \mathrm{~nm}$, we have $\xi=3.4 \mathrm{~nm}$ through $1 / \xi_{\text {expt }}=1 / l+1 / \xi$. Thus the correction factor $(1+\xi / l)$ yields 1.1 , giving a $10 \%$ upward correction to $n_{\mathrm{s}} / \mathrm{m}^{*}$; then, $\lambda_{\mathrm{L}}(0)=470-500 \mathrm{~nm}$ at ambient pressure. Using the $1 / \lambda_{\mathrm{L}}^{2}$ value and interlayer spacing $d=15 \AA$, the phase stiffness $\hbar^{2} d / 4 \mu_{0} e^{2} \lambda_{\mathrm{L}}^{2}$, measuring the energy scale of the macroscopic phase coherence [42], yields $\sim 40 \mathrm{~K}$, somewhat higher than $T_{\mathrm{c}}$, but much less than those of conventional BCS superconductors. This feature is likely connected to enhanced superconducting fluctuations observed in the Nernst effect, magnetization, and NMR relaxation [43-47]. 

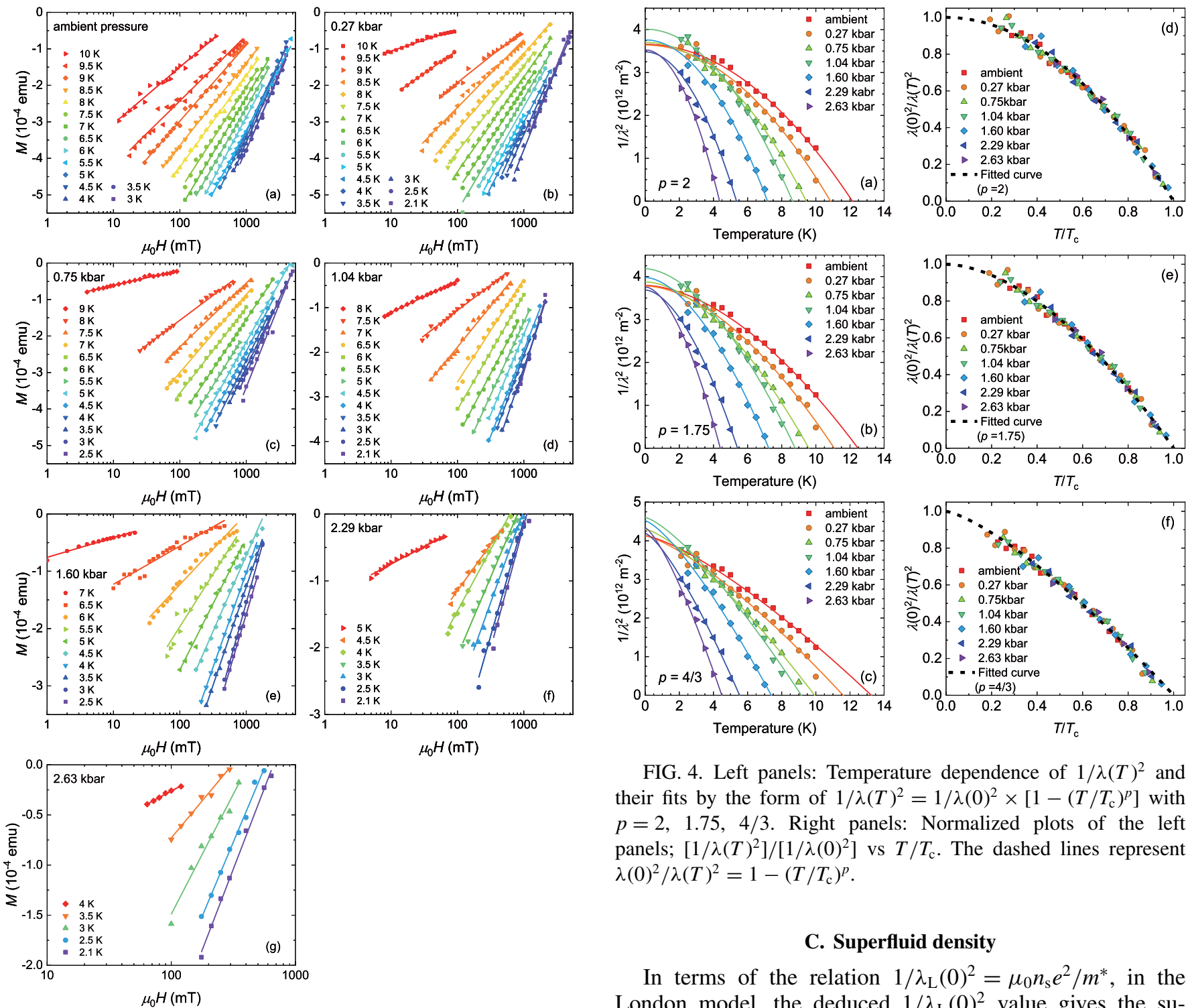

FIG. 4. Left panels: Temperature dependence of $1 / \lambda(T)^{2}$ and their fits by the form of $1 / \lambda(T)^{2}=1 / \lambda(0)^{2} \times\left[1-\left(T / T_{\mathrm{c}}\right)^{p}\right]$ with $p=2,1.75,4 / 3$. Right panels: Normalized plots of the left panels; $\left[1 / \lambda(T)^{2}\right] /\left[1 / \lambda(0)^{2}\right]$ vs $T / T_{\mathrm{c}}$. The dashed lines represent $\lambda(0)^{2} / \lambda(T)^{2}=1-\left(T / T_{\mathrm{c}}\right)^{p}$.

\section{Superfluid density}

In terms of the relation $1 / \lambda_{\mathrm{L}}(0)^{2}=\mu_{0} n_{\mathrm{S}} e^{2} / \mathrm{m}^{*}$, in the London model, the deduced $1 / \lambda_{\mathrm{L}}(0)^{2}$ value gives the superfluid density $n_{\mathrm{s}}(0)$ at every pressure. Figure $6(\mathrm{~b})$ shows the pressure dependence of $n_{\mathrm{s}}(0)$ per (BEDT-TTF $)_{2}$. The $n_{\mathrm{s}}(0)$ at ambient pressure is $0.62-0.70 /(\mathrm{BEDT}-\mathrm{TTF})_{2}(8-9 \times$ of the penetration depth.

In estimating $1 / \lambda_{\mathrm{L}}(0)^{2}$ under pressure, we have to take into consideration the pressure dependence of the correction factor $(1+\xi / l)$, because $\xi$ would vary with pressure while $l$ is supposed to stay constant. The relations $\xi=a v_{\mathrm{F}} / k_{\mathrm{B}} T_{\mathrm{c}}$ ( $k_{\mathrm{B}}$ : Boltzmann constant; $v_{\mathrm{F}}$ : Fermi velocity; $a$ : constant) and $v_{\mathrm{F}} \propto 1 / m^{*}$ jointly suggest that $\xi$ is inversely proportional to $m^{*} T_{\mathrm{c}}$, which varies with pressure. Referring to the pressure dependences of $T_{\mathrm{c}}$ shown in Fig. 1(c) and of the $\beta$-orbit (surrounding the whole Fermi surface) mass $m^{*}$, determined by the Shubnikov-de Haas oscillations [48], the pressure dependence of $(1+\xi / l)$ is calculated as shown in Fig. 5. Figure 6(a) displays $1 / \lambda_{\mathrm{L}}(0)^{2}\left[=(1+\xi / l) / \lambda(0)^{2}\right]$ against pressure, which shows a slightly upward trend towards higher pressures. This behavior of $1 / \lambda_{\mathrm{L}}(0)^{2}\left(\propto n_{\mathrm{s}} / m^{*}\right)$ differs from those of cuprates with overdoping and $\kappa$-NCS under pressure, in which the $n_{\mathrm{s}} / m^{*}$ values are reduced when carrier doped or pressurized, respectively.

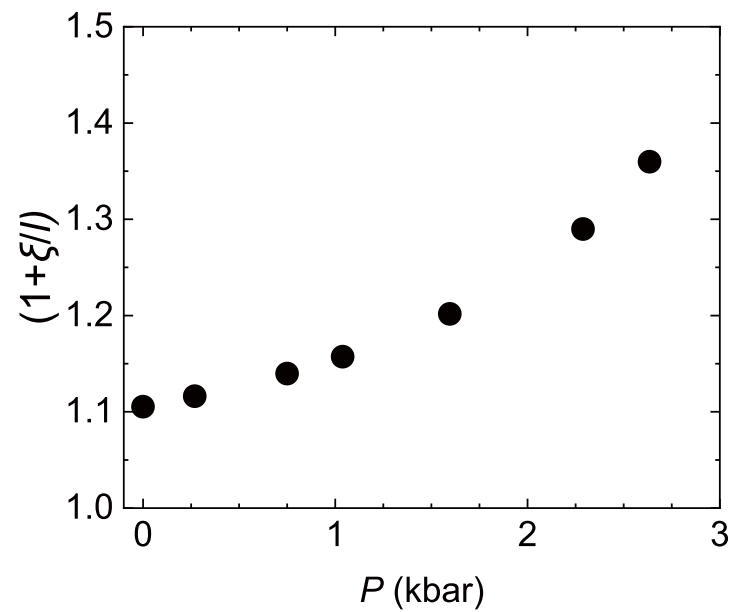

FIG. 5. Pressure dependence of the correction factor $(1+\xi / l)$. 

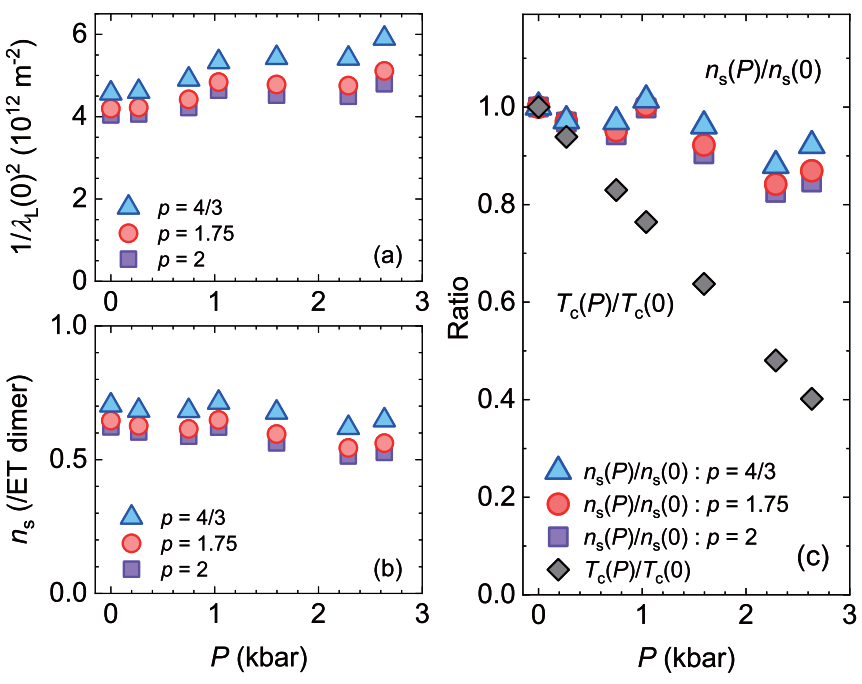

FIG. 6. (a) $1 / \lambda_{\mathrm{L}}(0)^{2}$ and (b) $n_{\mathrm{s}}(0)$ vs pressure. (c) Relative pressure dependences of $1 / \lambda_{\mathrm{L}}(0)^{2}, n_{\mathrm{s}}(0)$, and $T_{\mathrm{c}}$. The purple squares, red circles, and blue triangles show the $n_{\mathrm{s}}(0)$ values deduced from the fitting with $p=2,1.75$, and $4 / 3$, respectively, and the gray diamonds show $T_{\mathrm{c}}$. These values are normalized to the ambient-pressure values.

$10^{20} / \mathrm{cm}^{3}$ ), which is close to the normal-state carrier density $n_{\mathrm{n}}$ of $0.87 /(\text { BEDT-TTF })_{2}\left(1.0 \times 10^{21} / \mathrm{cm}^{3}\right)$ in the Hall coefficient measurements [49] or one hole per (BEDT-TTF) $)_{2}$. Most highlighted in the present results is that $n_{\mathrm{s}}(0)$ shows no meaningful pressure dependence, only a $8 \%-15 \%$ reduction at $2.6 \mathrm{kbar}$, whereas $T_{\mathrm{c}}$ is reduced by $60 \%$ at the pressure [Fig. 6(c)]. The significant reduction in $T_{\mathrm{c}}$ is considered to stem from the widening of the band leading to a suppression of electron correlation and/or a reduction of the density of states. Our observation indicates that nothing unusual happens in $n_{\mathrm{s}}(0)$ by pressure unlike in $T_{\mathrm{c}}$.

The present results differ from the previous reports on $\kappa$-NCS [21], which indicated a reduction in the superfluid density by pressure. Although this discrepancy may suggest that the superfluid issue be treated material by material, we note an experimental point to be cautious about particularly in experiments under pressure, that is, homogeneity in superconductivity. The magnitude and temperature dependence of magnetization, based on which the penetration depth is deduced, is influenced by the superconductive homogeneity. The present experiments ensured that the superconductive homogeneity is not deteriorated under pressure, in that (i) the superconducting transition probed by the ac susceptibility remains sharp even under pressure as shown in Fig. 1(b) (note that pressure inhomogeneity would make the transition broadened because $T_{\mathrm{c}}$ is sensitive to pressure) and (ii) the temperature dependence of $1 / \lambda(T)^{2}$ show a pressure-independent form, which is well approximated by widely accepted empirical forms as shown in Fig. 4. We note that this most crucial concern about experiments under pressure is overcome in the present experiments.

\section{CONCLUDING REMARKS}

The superconductivities in cuprates and organics share essential features such as their emergence from the mother phases, Mott insulators, and unconventional $d$-wave pairing. Thus, it may be a natural view that the heavy doping in cuprates and the application of pressure to organics, both working to weaken the electron correlation, have similar impacts on the superfluid density. However, the present study finds that, unlike the case of cuprates, the band broadening or the reduction of electron correlation in organics causes no appreciable change in the superfluid density, in sharp contrast to its great impact on the suppression of $T_{\mathrm{c}}$. As far as the problem of the missing superfluid density is concerned, the doping and bandwidth variation likely require distinctive considerations.

\section{ACKNOWLEDGMENTS}

The authors thank Cryogenic Research Center, University of Tokyo, for supporting low-temperature experiments. This work was supported in part by the Japan Society for the Promotion of Science (JSPS) Grants-in-Aid for Scientific Research (Grants No. JP19H01846 and No. JP18H05225) and the Murata Scientific Foundation.
[1] J. Bardeen, L. N. Cooper, and J. R. Schrieffer, Phys. Rev. 108, 1175 (1957).

[2] B. Keimer, S. A. Kivelson, M. R. Norman, S. Uchida, and J. Zaanen, Nature (London) 518, 179 (2015).

[3] Y. J. Uemura, G. M. Luke, B. J. Sternlieb, J. H. Brewer, J. F. Carolan, W. N. Hardy, R. Kadono, J. R. Kempton, R. F. Kiefl, S. R. Kreitzman, P. Mulhern, T. M. Riseman, D. L. Williams, B. X. Yang, S. Uchida, H. Takagi, J. Gopalakrishnan, A. W. Sleight, M. A. Subramanian, C. L. Chien et al., Phys. Rev. Lett. 62, 2317 (1989)

[4] Y. J. Uemura, A. Keren, L. P. Le, G. M. Luke, W. D. Wu, Y. Kubo, T. Manako, Y. Shimakawa, M. Subramanian, J. L. Cobb, and J. T. Markert, Nature (London) 364, 605 (1993).

[5] C. Niedermayer, C. Bernhard, U. Binninger, H. Glückler, J. L. Tallon, E. J. Ansaldo, and J. I. Budnick, Phys. Rev. Lett. 71, 1764 (1993).
[6] J.-P. Locquet, Y. Jaccard, A. Cretton, E. J. Williams, F. Arrouy, E. Mächler, T. Schneider, O. Fischer, and P. Martinoli, Phys. Rev. B 54, 7481 (1996).

[7] C. Bernhard, J. L. Tallon, T. Blasius, A. Golnik, and C. Niedermayer, Phys. Rev. Lett. 86, 1614 (2001).

[8] C. Panagopoulos, T. Xiang, W. Anukool, J. R. Cooper, Y. S. Wang, and C. W. Chu, Phys. Rev. B 67, 220502(R) (2003).

[9] T. R. Lemberger, I. Hetel, A. Tsukada, M. Naito, and M. Randeria, Phys. Rev. B 83, 140507(R) (2011).

[10] I. Bozovic, X. He, J. Wu, and A. T. Bollinger, Nature (London) 536, 309 (2016).

[11] F. Mahmood, X. He, I. Božović, and N. P. Armitage, Phys. Rev. Lett. 122, 027003 (2019).

[12] F. Kagawa, K. Miyagawa, and K. Kanoda, Nature (London) 436, 534 (2005).

[13] K. Kanoda, Hyperfine Interact. 104, 235 (1997). 
[14] K. Kanoda, J. Phys. Soc. Jpn. 75, 051007 (2006).

[15] K. Kanoda, Physica C 282-287, 299 (1997).

[16] A. Ardavan, S. Brown, S. Kagoshima, K. Kanoda, K. Kuroki, H. Mori, M. Ogata, S. Uji, and J. Wosnitza, J. Phys. Soc. Jpn. 81, 011004 (2012).

[17] R. T. Clay and S. Mazumdar, Phys. Rep. 788, 1 (2019).

[18] B. J. Powell and R. H. McKenzie, Rep. Prog. Phys. 74, 056501 (2011).

[19] P. A. Lee, Rep. Prog. Phys. 71, 012501 (2008).

[20] B. J. Powell and R. H. McKenzie, J. Phys.: Condens. Matter 16, L367 (2004).

[21] M. I. Larkin, A. Kinkhabwala, Y. J. Uemura, Y. Sushko, and G. Saito, Phys. Rev. B 64, 144514 (2001).

[22] A. M. Kini, U. Geiser, H. H. Wang, K. D. Carlson, J. M. Williams, W. K. Kwok, K. G. Vandervoort, J. E. Thompson, and D. L. Stupka, Inorg. Chem. 29, 2555 (1990).

[23] Y. Uwatoko, T. Hotta, E. Matsuoka, H. Mori, T. Ohki, J. L. Sarraot, J. D. Thompsont, N. Möri, and G. Oomi, Rev. High Pressure Sci. Technol. 7, 1508 (1998).

[24] J. E. Schirber, D. L. Overmyer, J. M. Williams, A. M. Kini, and H. H. Wang, Physica C 170, 231 (1990).

[25] C. Strack, C. Akinci, V. Paschenko, B. Wolf, E. Uhrig, W. Assmus, M. Lang, J. Schreuer, L. Wiehl, J. A. Schlueter, J. Wosnitza, D. Schweitzer, J. Müller, and J. Wykhoff, Phys. Rev. B 72, 054511 (2005).

[26] Y. V. Sushko, V. A. Bondarenko, R. A. Petrosov, N. D. Kushch, and E. B. Yagubskii, J. Phys. I France 1, 1375 (1991).

[27] X. Su, F. Zuo, J. A. Schlueter, M. E. Kelly, and J. M. Williams, Phys. Rev. B 57, R14056(R) (1998).

[28] T. F. Stalcup, J. S. Brooks, and R. C. Haddon, Phys. Rev. B 60, 9309 (1999).

[29] N. Yoneyama, A. Higashihara, T. Sasaki, T. Nojima, and N. Kobayashi, J. Phys. Soc. Jpn. 73, 1290 (2004).

[30] Y. Ait Ahmed, A. Tirbiyine, D. Soubane, A. Taoufik, H. El Ouaddi, H. El hamidi, A. Abou Alhassan, A. Hafid, and S. Senoussi, Mater. Today Proc. 24, 100 (2020).

[31] P. G. de Gennes, Superconductivity of Metals and Alloys (Benjamin, New York, 1966).

[32] Z. Hao and J. R. Clem, Phys. Rev. Lett. 67, 2371 (1991).
[33] A. J. Schultz, H. H. Wang, J. M. Williams, L. W. Finger, R. M. Hazen, C. Rovira, and M.-H. Whangbo, Physica C 234, 300 (1994).

[34] L. H. Lewis and K. M. Bussmann, Rev. Sci. Instrum. 67, 3537 (1996).

[35] R. Prozorov and R. W. Giannetta, Supercond. Sci. Technol. 19, R41 (2006).

[36] M. Lang, N. Toyota, T. Sasaki, and H. Sato, Phys. Rev. B 46, 5822 (1992).

[37] M. Tinkham, Introduction to Superconductivity, 2nd ed. (McGraw-Hill, New York, 1996).

[38] H. Weiss, M. V. Kartsovnik, W. Biberacher, E. Steep, A. G. M. Jansen, and N. D. Kushch, JETP Lett. 66, 202 (1997).

[39] C. H. Mielke, N. Harrison, D. G. Rickel, A. H. Lacerda, R. M. Vestal, and L. K. Montgomery, Phys. Rev. B 56, R4309(R) (1997).

[40] W. K. Kwok, U. Welp, K. D. Carlson, G. W. Crabtree, K. G. Vandervoort, H. H. Wang, A. M. Kini, J. M. Williams, D. L. Stupka, L. K. Montgomery, and J. E. Thompson, Phys. Rev. B 42, 8686 (1990).

[41] H. Ito, M. Watanabe, Y. Nogami, T. Ishiguro, T. Komatsu, G. Saito, and N. Hosoito, J. Phys. Soc. Jpn. 60, 3230 (1991).

[42] V. J. Emery and S. A. Kivelson, Nature (London) 374, 434 (1995).

[43] M. Lang, F. Steglich, N. Toyota, and T. Sasaki, Phys. Rev. B 49, 15227 (1994).

[44] M.-S. Nam, A. Ardavan, S. J. Blundell, and J. A. Schlueter, Nature (London) 449, 584 (2007).

[45] S. Tsuchiya, J. Yamada, T. Terashima, N. Kurita, K. Kodama, K. Sugii, and S. Uji, J. Phys. Soc. Jpn. 82, 064711 (2013).

[46] T. Uehara, M. Ito, H. Taniguchi, and K. Satoh, J. Phys. Soc. Jpn. 82, 073706 (2013).

[47] T. Kobayashi, Y. Ihara, Y. Saito, and A. Kawamoto, Phys. Rev. B 89, 165141 (2014).

[48] H. Weiss, M. V. Kartsovnik, W. Biberacher, E. Steep, E. Balthes, A. G. M. Jansen, K. Andres, and N. D. Kushch, Phys. Rev. B 59, 12370 (1999).

[49] K. Katayama, T. Nagai, H. Taniguchi, K. Satoh, N. Tajima, and R. Kato, J. Phys. Soc. Jpn. 76, 194 (2007). 